\title{
On some sufficient conditions for univalence and starlikeness
}

Janusz Sokół ${ }^{*}$ and Mamoru Nunokawa²

"Correspondence: jsokol@prz.edu.pl ${ }^{1}$ Department of Mathematics, Rzeszów University of Technology, Al. Powstańców Warszawy 12, Rzeszów, 35-959, Poland Full list of author information is available at the end of the article

\section{Abstract}

In this work, the conditions for univalence, starlikeness and convexity are discussed.

MSC: Primary 30C45; secondary 30C80

Keywords: strongly starlike functions; convex functions of order alpha; Jack's lemma; Nunokawa's lemma; Umezawa condition; univalence criteria

\section{Introduction}

We shall consider the set $\mathcal{H}$ of all analytic functions in the open unit disc

$$
\mathbb{D}=\{z:|z|<1\}
$$

on the complex plane $\mathbb{C}$ and

$$
\mathcal{A}=\left\{f \in \mathcal{H}: f(z)=z+a_{2} z^{2}+\cdots\right\} .
$$

The class $\mathcal{S}_{\alpha}^{*}$ of starlike functions of order $\alpha<1$ may be defined as

$$
\mathcal{S}_{\alpha}^{*}=\left\{f \in \mathcal{A}: \mathfrak{R e} \frac{z f^{\prime}(z)}{f(z)}>\alpha, z \in \mathbb{D}\right\} .
$$

The class $\mathcal{S}_{\alpha}^{*}$ and the class $\mathcal{K}_{\alpha}$ of convex functions of order $\alpha<1$

$$
\begin{aligned}
\mathcal{K}_{\alpha} & :=\left\{f \in \mathcal{A}: \mathfrak{R e}\left(1+\frac{z f^{\prime \prime}(z)}{f^{\prime}(z)}\right)>\alpha, z \in \mathbb{D}\right\} \\
& =\left\{f \in \mathcal{A}: z f^{\prime} \in \mathcal{S}_{\alpha}^{*}\right\}
\end{aligned}
$$

were introduced by Robertson in [1]. If $\alpha \in[0 ; 1)$, then a function in either of these sets is univalent. In particular, we denote by $\mathcal{S}_{0}^{*}=\mathcal{S}^{*}, \mathcal{K}_{0}=\mathcal{K}$ the classes of starlike and convex functions, respectively.

\section{Preliminaries}

Lemma 2.1 Let $O=0, P=\alpha+i a \alpha, Q=x+i a x$ and $A \in(0,+\infty)$ be the points on the complex plane, where $0<\alpha \leq A / 2, \alpha<x$ and $-\infty<a<\infty$. Then we have

$$
\frac{|A-Q|}{|O-Q|}<\frac{|A-P|}{|O-P|} \leq \frac{A-\alpha}{\alpha} .
$$

\section{Springer}

- 2012 Sokół and Nunokawa; licensee Springer. This is an Open Access article distributed under the terms of the Creative Commons Attribution License (http://creativecommons.org/licenses/by/2.0), which permits unrestricted use, distribution, and reproduction in any medium, provided the original work is properly cited. 


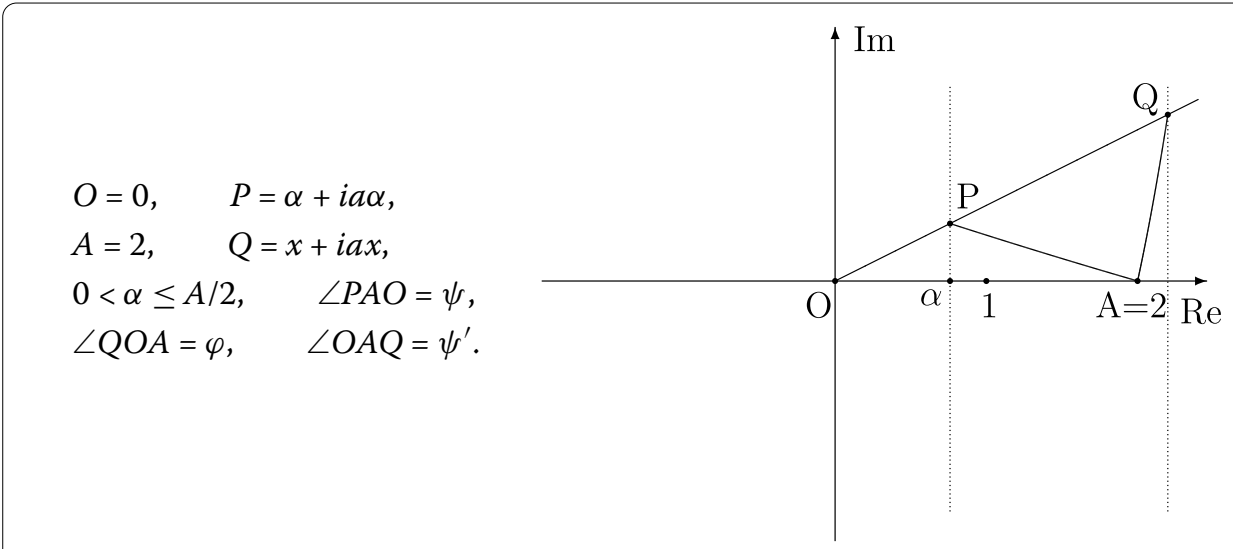

Figure 1 The lines and the points.

Proof For $a=0$, the assertion is obvious. For $a \neq 0$, consider the triangles $O A P$ and $O A Q$, see Figure 1. Both of them have the same angle $\varphi$ at the point $O$. Let the first have the angle $\psi$ at the point $A$ and the second have the angle $\psi^{\prime}$ at the point $A$. Then the hypothesis $0<\alpha \leq A / 2$ implies $\cos \varphi \leq \cos \psi$. Further we have

$$
\cos \varphi=\frac{\alpha}{|P|}, \quad \cos \psi=\frac{A-\alpha}{|A-P|} .
$$

This gives the second inequality of the assertion. The first one follows immediately from $\sin \psi^{\prime}>\sin \psi$ and

$$
\frac{|A-P|}{|P|}=\frac{\sin \varphi}{\sin \psi}, \quad \frac{|A-Q|}{|Q|}=\frac{\sin \varphi}{\sin \psi^{\prime}} .
$$

\section{Main result}

Theorem 3.1 Let $p(z)=1+\sum_{n=k \geq 1}^{\infty} c_{n} z^{n}$ be analytic in the unit disc $\mathbb{D}$ and $\alpha$ be a positive real number $0<\alpha \leq 1 / 2$. Then suppose that there exists a point $z_{0},\left|z_{0}\right|<1$ such that

$$
\mathfrak{R e}\{p(z)\}>\alpha \text { for }|z|<\left|z_{0}\right|
$$

and

$$
\mathfrak{R e}\left\{p\left(z_{0}\right)\right\}=p\left(z_{0}\right)=\alpha .
$$

Then we have

$$
\frac{z_{0} p^{\prime}\left(z_{0}\right)}{p\left(z_{0}\right)} \leq-k(1-\alpha)
$$

Proof Let us put

$$
q(z)=\frac{p(z)-1}{p(z)}, \quad q(0)=0 .
$$


Then the function $q$ is analytic in $|z| \leq\left|z_{0}\right|<1$ and from the hypothesis of Theorem 3.1 and Lemma 2.1, with $A=1$, we have

$$
|q(z)|<\frac{1-\alpha}{\alpha}
$$

for $|z| \leq\left|z_{0}\right|$ and

$$
\left|q\left(z_{0}\right)\right|=\frac{1-\alpha}{\alpha} .
$$

This shows that $|q(z)|$ takes its maximum at $z=z_{0}$ on the circle $|z|=\left|z_{0}\right|$. Then from FukuiSakaguchi [2] and Jack's [3] lemmas, there exists a real number $k \geq 1$ such that

$$
\begin{aligned}
\frac{z_{0} q^{\prime}\left(z_{0}\right)}{q\left(z_{0}\right)} & =\frac{z_{0} p^{\prime}\left(z_{0}\right)}{p\left(z_{0}\right)-1}-\frac{z_{0} p^{\prime}\left(z_{0}\right)}{p\left(z_{0}\right)} \\
& =z_{0} p^{\prime}\left(z_{0}\right)\left(\frac{1}{\alpha-1}-\frac{1}{\alpha}\right) \\
& =\frac{z_{0} p^{\prime}\left(z_{0}\right)}{\alpha(\alpha-1)} \\
& \geq k .
\end{aligned}
$$

This shows that $z_{0} p^{\prime}\left(z_{0}\right)$ is a negative real number and

$$
\frac{z_{0} p^{\prime}\left(z_{0}\right)}{\alpha}=\frac{z_{0} p^{\prime}\left(z_{0}\right)}{p\left(z_{0}\right)} \leq-k(1-\alpha)
$$

This completes the proof of Theorem 3.1.

Theorem 3.1 is, in a certain sense, the supplement of Nunokawa's lemma [4]. From Theorem 3.1 we have the following corollaries.

Corollary 3.2 Let $p(z)=1+\sum_{n=1}^{\infty} c_{n} z^{n}$ be analytic in the unit disc $\mathbb{D}$ and $\alpha$ be a positive real number $0<\alpha \leq 1 / 2$. Suppose also that for arbitrary $r, 0<r<1$, $p$ satisfies the condition

$$
\min _{|z| \leq r} \mathfrak{R e}\{p(z)\}=\min _{|z| \leq r}|p(z)|
$$

and

$$
\mathfrak{R e}\left\{p(z)+\frac{z p^{\prime}(z)}{p(z)}\right\}>2 \alpha-1 \quad \text { for }|z|<1
$$

Then we have

$$
\mathfrak{R e}\{p(z)\}>\alpha \quad \text { for }|z|<1 .
$$

Proof If there exists a point $z_{0},\left|z_{0}\right|<1$, such that

$$
\mathfrak{R e}\{p(z)\}>\alpha \text { for }|z|<\left|z_{0}\right|
$$


and

$$
\mathfrak{R e}\left\{p\left(z_{0}\right)\right\}=\alpha, \quad 0<\alpha \leq 1 / 2,
$$

then from the hypothesis of Corollary 3.2, we have

$$
\mathfrak{R e}\left\{p\left(z_{0}\right)\right\}=p\left(z_{0}\right)=\alpha
$$

Then from Theorem 3.1, we have

$$
\frac{z_{0} p^{\prime}\left(z_{0}\right)}{p\left(z_{0}\right)} \leq \alpha-1
$$

and therefore we have

$$
\mathfrak{R e}\left\{p\left(z_{0}\right)+\frac{z_{0} p^{\prime}\left(z_{0}\right)}{p\left(z_{0}\right)}\right\} \leq 2 \alpha-1 .
$$

This contradicts the hypothesis of Corollary 3.2 and it completes the proof of Corollary 3.2.

Corollary 3.3 Let $f(z)=z+\sum_{n=2}^{\infty} a_{n} z^{n}$ be analytic in the unit disc $\mathbb{D}$ and $\alpha$ be a positive real number $0<\alpha \leq 1 / 2$. Suppose that for arbitrary $r, 0<r<1, f$ satisfies the condition

$$
\min _{|z| \leq r} \mathfrak{R e}\left\{\frac{z f^{\prime}(z)}{f(z)}\right\}=\min _{|z| \leq r}\left|\frac{z f^{\prime}(z)}{f(z)}\right|
$$

and

$$
\mathfrak{R e}\left\{1+\frac{z f^{\prime \prime}(z)}{f^{\prime}(z)}\right\}>2 \alpha-1 \quad \text { for }|z|<1,
$$

where

$$
-1<2 \alpha-1 \leq 0 .
$$

Then we have

$$
\mathfrak{R e}\left\{\frac{z f^{\prime}(z)}{f(z)}\right\}>\alpha \quad \text { for }|z|<1,
$$

orf is starlike of order $\alpha$.

Proof Putting

$$
p(z)=\frac{z f^{\prime}(z)}{f(z)},
$$

it follows that

$$
p(z)+\frac{z p^{\prime}(z)}{p(z)}=1+\frac{z f^{\prime \prime}(z)}{f^{\prime}(z)} .
$$

Then from Corollary 3.2, we have (3.9). 
Theorem 3.4 Let $p(z)=1+\sum_{n=k \geq 1}^{\infty} c_{n} z^{n}$ be analytic in the unit disc $\mathbb{D}$ and $\alpha$ be a positive real number $1 / 2<\alpha<1$. Then suppose that there exists a point $z_{0} \in \mathbb{D}$ such that

$$
\mathfrak{R e}\{p(z)\}>\alpha \text { for }|z|<\left|z_{0}\right|
$$

and

$$
\mathfrak{R e}\left\{p\left(z_{0}\right)\right\}=p\left(z_{0}\right)=\alpha .
$$

Then we have

$$
\frac{z_{0} p^{\prime}\left(z_{0}\right)}{p\left(z_{0}\right)}=\mathfrak{R e}\left\{\frac{z_{0} p^{\prime}\left(z_{0}\right)}{p\left(z_{0}\right)}\right\} \leq-\frac{k(2-\alpha)}{2} .
$$

Proof Let us put

$$
q(z)=\frac{2-p(z)}{p(z)}-1, \quad q(0)=0 .
$$

Then from Lemma 2.1, with $A=2$, we have that $|q(z)+1|$ takes its maximum value at $z=z_{0}$ on the circle $|z|=\left|z_{0}\right|$ or

$$
\max _{|z|=\left|z_{0}\right|}|q(z)+1|=\max _{|z|=\left|z_{0}\right|}\left|\frac{2-p(z)}{p(z)}\right|=\frac{2-\alpha}{\alpha} .
$$

Applying Jack [3], Miller-Mocanu [5] and Fukui-Sakaguchi's [2] lemmas, there exists a real number $m \geq k$ such that

$$
\begin{aligned}
\frac{z_{0} q^{\prime}\left(z_{0}\right)}{q\left(z_{0}\right)} & =-\frac{z_{0} p^{\prime}\left(z_{0}\right)}{2-p\left(z_{0}\right)}-\frac{z_{0} p^{\prime}\left(z_{0}\right)}{p\left(z_{0}\right)} \\
& =-z_{0} p^{\prime}\left(z_{0}\right)\left(\frac{1}{2-\alpha}+\frac{1}{\alpha}\right) \\
& =-\frac{2 z_{0} p^{\prime}\left(z_{0}\right)}{\alpha(2-\alpha)} \\
& =-\frac{z_{0} p^{\prime}\left(z_{0}\right)}{p\left(z_{0}\right)}\left(\frac{2}{2-\alpha}\right) \\
& \geq m \geq k .
\end{aligned}
$$

This shows that

$$
\frac{z_{0} p^{\prime}\left(z_{0}\right)}{p\left(z_{0}\right)} \leq-\frac{k(2-\alpha)}{2}
$$

This completes the proof of Theorem 3.4.

Corollary 3.5 Let $f(z)=z+\sum_{n=2}^{\infty} a_{n} z^{n}$ be analytic in the unit disc $\mathbb{D}$ and $\alpha$ be a positive real number $1 / 2<\alpha<1$. Suppose that for arbitrary $r, 0<r<1, f$ satisfies the condition

$$
\min _{|z| \leq r} \mathfrak{R e}\left\{\frac{z f^{\prime}(z)}{f(z)}\right\}=\min _{|z| \leq r}\left|\frac{z f^{\prime}(z)}{f(z)}\right|
$$


and

$$
\mathfrak{R e}\left\{1+\frac{z f^{\prime \prime}(z)}{f^{\prime}(z)}\right\}>\frac{3 \alpha-2}{2} \quad \text { for }|z|<1 .
$$

Then we have

$$
\mathfrak{R e}\left\{\frac{z f^{\prime}(z)}{f(z)}\right\}>\alpha \quad \text { for }|z|<1
$$

Proof Applying the same method as in the proof of Corollary 3.3 and in the proof of Lemma 2.1, we can obtain Corollary 3.5.

Corollary 3.6 Let $F(z)=1 / z+\sum_{n=1}^{\infty} b_{n} z^{n}$ be analytic and not vanishing in the punctured unit disc $0<|z|<1$ and let $\alpha$ be a positive real number $0<\alpha<1 / 2$. Suppose also that for arbitrary $r, 0<r<1, F$ satisfies the following condition:

$$
\min _{|z| \leq r} \mathfrak{R e}\left\{-\frac{z F^{\prime}(z)}{F(z)}\right\}=\min _{|z| \leq r}\left|\frac{z F^{\prime}(z)}{F(z)}\right|
$$

and

$$
-\mathfrak{R e}\left\{1+\frac{z F^{\prime \prime}(z)}{F^{\prime}(z)}\right\}<2-\alpha \quad \text { for }|z|<1 .
$$

Then we have

$$
\mathfrak{R e}\left\{-\frac{z F^{\prime}(z)}{F(z)}\right\}>\alpha \quad \text { for }|z|<1
$$

Proof Putting

$$
p(z)=-\frac{z F^{\prime}(z)}{F(z)}=-\frac{z\left(-1 / z^{2}+\sum_{n=1}^{\infty} n b_{n} z^{n-1}\right)}{1 / z+\sum_{n=1}^{\infty} b_{n} z^{n}}
$$

then we have

$$
p(z)=1+\sum_{n=2}^{\infty} c_{n} z^{n}, \quad p(0)=1
$$

and it follows that

$$
p(z)-\frac{z p^{\prime}(z)}{p(z)}=-\left(1+\frac{z F^{\prime \prime}(z)}{F^{\prime}(z)}\right) .
$$

If there exists a point $z_{0} \in \mathbb{D}$ such that

$$
\mathfrak{R e}\{p(z)\}>\alpha \text { for }|z|<\left|z_{0}\right|
$$

and

$$
\mathfrak{R e}\left\{p\left(z_{0}\right)\right\}=p\left(z_{0}\right)=\alpha,
$$


then from the hypothesis, we have $p\left(z_{0}\right)=\alpha$. Applying Theorem 3.1, we have

$$
\frac{z_{0} p^{\prime}\left(z_{0}\right)}{p\left(z_{0}\right)} \leq-2(1-\alpha)
$$

and therefore we have

$$
\begin{aligned}
\mathfrak{R e}\left\{p\left(z_{0}\right)-\frac{z_{0} p^{\prime}\left(z_{0}\right)}{p\left(z_{0}\right)}\right\} & =-\mathfrak{R e}\left\{1+\frac{z_{0} F^{\prime \prime}\left(z_{0}\right)}{F^{\prime}\left(z_{0}\right)}\right\} \\
& \geq \alpha+2(1-\alpha) \\
& =2-\alpha .
\end{aligned}
$$

This contradicts the hypothesis and therefore we have

$$
\mathfrak{R e}\{p(z)\}=\mathfrak{R e}\left\{-\frac{z F^{\prime}(z)}{F(z)}\right\}>\alpha \quad \text { for }|z|<1,
$$

and this shows that $F$ is meromorphic starlike of order $\alpha$ in the punctured unit disc $0<$ $|z|<1$.

We note the following interesting result which was published in a minor journal and so it was not well known in the public of univalent function theory but is strongly connected with the previous Corollaries 3.3, 3.5 and 3.6.

Lemma 3.7 ([6]) Let $f(z)=z+a_{2} z^{2}+\cdots$ be an analytic function in $|z|<1$ with $f(z) f^{\prime}(z) / z \neq$ 0 in $|z|<1$. Then, for each $\alpha,-1 / 2<\alpha<0$, there exists a function $f$ which satisfies

$$
1+\mathfrak{R e} \frac{z f^{\prime \prime}(z)}{f^{\prime}(z)}>\alpha \quad \text { in }|z|<1
$$

butf is not starlike in $|z|<1$.

In [6] the authors pointed out that the function

$$
f(z)=\frac{(1-z)^{2 \alpha-1}-1}{1-2 \alpha}
$$

satisfies the above conditions.

Lemma 3.8 Let $p(z)=1+\sum_{n=m \geq 2}^{\infty} c_{n} z^{n}$ be an analytic function in $\mathbb{D}$. Suppose also that there exists a point $z_{0} \in \mathbb{D}$ such that

$$
\mathfrak{R e}\{p(z)\}>0 \text { for }|z|<\left|z_{0}\right|
$$

and

$$
\mathfrak{R e}\left\{p\left(z_{0}\right)\right\}=0 \text { and } p\left(z_{0}\right) \neq 0 \text {. }
$$


Then we have

$$
\frac{z_{0} p^{\prime}\left(z_{0}\right)}{p\left(z_{0}\right)}=i k
$$

where $k$ is a real number and

$$
k \geq \frac{m}{2}\left(a+\frac{1}{a}\right) \geq m \geq 2 \quad \text { when } \arg p\left(z_{0}\right)=\frac{\pi}{2}
$$

and

$$
k \leq-\frac{m}{2}\left(a+\frac{1}{a}\right) \leq-m \leq-2 \quad \text { when } \arg p\left(z_{0}\right)=-\frac{\pi}{2},
$$

where

$$
p\left(z_{0}\right)= \pm i a \text { and } a>0 \text {. }
$$

Proof Let us put

$$
\phi(z)=\frac{1-p(z)}{1+p(z)}, \quad|z|<1
$$

Then we have $\phi(0)=\phi^{\prime}(0)=\cdots=\phi^{(m-1)}(0)=0,|\phi(z)|<1$, for $|z|<\left|z_{0}\right|$ and $\left|\phi\left(z_{0}\right)\right|=1$. From $[2,3]$ and $[5]$, we obtain

$$
\frac{z_{0} \phi^{\prime}\left(z_{0}\right)}{\phi\left(z_{0}\right)}=-\frac{2 z_{0} p^{\prime}\left(z_{0}\right)}{1-p^{2}\left(z_{0}\right)}=-\frac{2 z_{0} p^{\prime}\left(z_{0}\right)}{1+\left|p\left(z_{0}\right)\right|^{2}} \geq m .
$$

This shows that

$$
-z_{0} p^{\prime}\left(z_{0}\right) \geq \frac{m}{2}\left(1+\left|p\left(z_{0}\right)\right|^{2}\right)
$$

and $z_{0} p^{\prime}\left(z_{0}\right)$ is a negative real number. For the case $\arg p\left(z_{0}\right)=\pi / 2, p\left(z_{0}\right)=i a$ and $0<a$, we have

$$
\mathfrak{R e}\left\{\frac{z_{0} p^{\prime}\left(z_{0}\right)}{p\left(z_{0}\right)}\right\}=\mathfrak{R e}\left\{\frac{z_{0} p^{\prime}\left(z_{0}\right)}{i a}\right\}=\mathfrak{R e}\left\{-\frac{i z_{0} p^{\prime}\left(z_{0}\right)}{a}\right\}=0
$$

and

$$
\mathfrak{I m}\left\{\frac{z_{0} p^{\prime}\left(z_{0}\right)}{p\left(z_{0}\right)}\right\}=\mathfrak{I m}\left\{-\frac{i z_{0} p^{\prime}\left(z_{0}\right)}{a}\right\} \geq \frac{m}{2}\left(\frac{1+\left|p\left(z_{0}\right)\right|^{2}}{a}\right)=\frac{m}{2}\left(a+\frac{1}{a}\right) \geq m
$$

For the case $\arg p\left(z_{0}\right)=-\pi / 2, p\left(z_{0}\right)=-i a$ and $0<a$, applying the same method as above, we have

$$
\mathfrak{R e}\left\{\frac{z_{0} p^{\prime}\left(z_{0}\right)}{p\left(z_{0}\right)}\right\}=0
$$


and

$$
\mathfrak{I m}\left\{\frac{z_{0} p^{\prime}\left(z_{0}\right)}{p\left(z_{0}\right)}\right\} \leq-\frac{m}{2}\left(a+\frac{1}{a}\right) \leq-m .
$$

This completes the proof.

Theorem 3.9 Let $f(z)=z+\sum_{n=m+1}^{\infty} a_{n} z^{n}$ be analytic in the unit disc $\mathbb{D}$. Suppose also that

$$
\mathfrak{R e}\left\{1+\frac{z f^{\prime \prime}(z)}{f^{\prime}(z)}\right\}<\frac{8+m}{6} \text { for }|z|<1
$$

where $1 \leq m$. If $z f^{\prime}(z) / f(z)$ is analytic in $\mathbb{D}$ and omits $4 / 3$, then $f$ is starlike in the unit disc $\mathbb{D}$.

Proof Let us put

$$
\frac{z f^{\prime}(z)}{f(z)}=\frac{4 p(z)}{1+3 p(z)}
$$

where $p(0)=1, p^{\prime}(0)=p^{\prime \prime}(0)=\cdots=p^{(m-1)}(0)=0$. Then it follows that

$$
1+\frac{z f^{\prime \prime}(z)}{f^{\prime}(z)}=\frac{z f^{\prime}(z)}{f(z)}+\frac{z p^{\prime}(z)}{p(z)}-\frac{3 z p^{\prime}(z)}{1+3 p(z)} .
$$

If there exists a point $z_{0} \in \mathbb{D}$ such that

$$
\mathfrak{R e}\{p(z)\}>0 \text { for }|z|<\left|z_{0}\right|
$$

and

$$
\mathfrak{R e}\left\{p\left(z_{0}\right)\right\}=0
$$

and $p\left(z_{0}\right) \neq 0$, because $p\left(z_{0}\right)=0$ contradicts the hypothesis (3.21), then from Lemma 3.8, we have

$$
\frac{z_{0} p^{\prime}\left(z_{0}\right)}{p\left(z_{0}\right)}=i k
$$

where $k$ is a real number and $m \leq|k|$. For the case $\arg p\left(z_{0}\right)=\pi / 2, p\left(z_{0}\right)=i a$ and $0<a$, we have

$$
\begin{aligned}
1+\frac{z_{0} f^{\prime \prime}\left(z_{0}\right)}{f^{\prime}\left(z_{0}\right)} & =\frac{z_{0} f^{\prime}\left(z_{0}\right)}{f\left(z_{0}\right)}+\frac{z_{0} p^{\prime}\left(z_{0}\right)}{p\left(z_{0}\right)}-\frac{3 z_{0} p^{\prime}\left(z_{0}\right)}{p\left(z_{0}\right)} \frac{p\left(z_{0}\right)}{1+3 p\left(z_{0}\right)} \\
& =\frac{4 i a}{1+3 i a}+i k-3 i k \frac{i a}{1+3 i a} \\
& =\frac{4 i a(1-3 i a)}{1+9 a^{2}}+i k+\frac{a k(1-3 i a)}{1+9 a^{2}} .
\end{aligned}
$$


Therefore, we have

$$
\begin{aligned}
\mathfrak{R e}\left\{1+\frac{z_{0} f^{\prime \prime}\left(z_{0}\right)}{f^{\prime}\left(z_{0}\right)}\right\} & =\frac{12 a^{2}}{1+9 a^{2}}+\frac{3 a k}{1+9 a^{2}} \\
& \geq \frac{12 a^{2}}{1+9 a^{2}}+\frac{3 m\left(1+a^{2}\right)}{2\left(1+9 a^{2}\right)} \\
& =\frac{24 a^{2}+3 m a^{2}+3 m}{2\left(1+9 a^{2}\right)} .
\end{aligned}
$$

Putting

$$
h(x)=\frac{(8+m) x^{2}+m}{1+9 x^{2}}, \quad x>0
$$

we have

$$
h^{\prime}(x)=\frac{16(1-m) x}{\left(1+9 x^{2}\right)^{2}}<0, \quad x>0 .
$$

This shows that

$$
\mathfrak{R e}\left\{1+\frac{z_{0} f^{\prime \prime}\left(z_{0}\right)}{f^{\prime}\left(z_{0}\right)}\right\} \geq \lim _{a \rightarrow \infty} \frac{24 a^{2}+3 m a^{2}+3 m}{2\left(1+9 a^{2}\right)}=\frac{24+3 m}{18} .
$$

This contradicts the hypothesis (3.21).

For the case $\arg p\left(z_{0}\right)=-\pi / 2, p\left(z_{0}\right)=-i a$ and $0<a$, applying the same method as above, we also have (3.22). This is also a contradiction and therefore it completes the proof of Theorem 3.9.

Remark 3.10 Singh and Singh obtained in [7] that if $f(z)=z+a_{2} z^{2}+\cdots$ is analytic in $\mathbb{D}$ and

$$
\mathfrak{R e}\left\{1+\frac{z f^{\prime \prime}(z)}{f^{\prime}(z)}\right\}<\frac{3}{2} \quad \text { for }|z|<1
$$

then $f$ is starlike in $\mathbb{D}$. Earlier, Ozaki [8] proved the univalence of $f$ in $\mathbb{D}$ under the same assumption (3.23).

For $m=1$, the inequality (3.21) becomes (3.23), so Theorem 3.9 is a generalization of the above result.

\section{Competing interests}

The authors declare that they have no competing interests.

Authors' contributions

All authors contributed equally and significantly in writing this paper. All authors read and approved the final manuscript.

\section{Author details}

'Department of Mathematics, Rzeszów University of Technology, Al. Powstańców Warszawy 12, Rzeszów, 35-959, Poland.

${ }^{2}$ University of Gunma, Hoshikuki-cho 798-8, Chuou-Ward, Chiba, 260-0808, Japan.

\section{Acknowledgements}

The authors sincerely thank the referees for pointing out a few corrections in the original draft of the paper. 


\section{References}

1. Robertson, MS: On the theory of univalent functions. Ann. Math. 37, 374-408 (1936)

2. Fukui, S, Sakaguchi, K: An extension of a theorem Ruscheweyh. Bull. Fac. Edu. Wakayama Univ. Nat. Sci. 29, 1-3 (1980)

3. Jack, IS: Functions starlike and convex of order $\alpha$. J. Lond. Math. Soc. 3, 469-474 (1971)

4. Nunokawa, M: On properties of non-Carathéodory functions. Proc. Jpn. Acad., Ser. A 68(6), 152-153 (1992)

5. Miller, SS, Mocanu, PT: Second order differential inequalities in the complex plane. J. Math. Anal. Appl. 65, 289-305 (1978)

6. Pfaltgraff, JA, Reade, MO, Umezawa, T: Sufficient conditions for univalence. Ann. Fac. Sci. Kinshasa, Sect. Math. Phys. 2, 94-100 (1976)

7. Singh, R, Singh, S: Some sufficient conditions for univalence and starlikeness. Colloq. Math. 47, 309-314 (1982)

8. Ozaki, S: On the theory of multivalent functions. Sci. Rep. Tokyo Bunrika Daigaku 4, 45-86 (1941)

doi:10.1186/1029-242X-2012-282

Cite this article as: Sokół and Nunokawa: On some sufficient conditions for univalence and starlikeness. Journal of Inequalities and Applications 2012 2012:282.

\section{Submit your manuscript to a SpringerOpen ${ }^{\circ}$ journal and benefit from:}

- Convenient online submission

- Rigorous peer review

- Immediate publication on acceptance

- Open access: articles freely available online

- High visibility within the field

- Retaining the copyright to your article 